${ }^{1}$ Institut für Arbeitsmedizin, Sozialmedizin und Umweltmedizin der Goethe-Universität Frankfurt am Main

\title{
Infektionen mit neuem humanen Betacoronavirus 2c EMC/2012 (hCoV-EMC)
}

Stefanie Uibel ${ }^{1}$, David A. Groneberg ${ }^{1}$

S. Uibel, D. A. Groneberg: Infektionen mit neuem humanen Betacoronavirus 2c EMC/2012 (hCoV-EMC). Zbl Arbeitsmed 63 (2013) 54-56

Schlüsselwörter: hCoV-EMC, Betacoronavirus 2c EMC/2012, humanes Coronavirus

Zusammenfassung:

Ein bis dato unbekanntes Coronavirus (hCoV-EMC) konnte Ende 2012 erstmals retrospektiv aus dem Sputum eines ansonsten gesunden 60jährigen Patienten aus Saudi-Arabien isoliert werden, welcher sich mit akutem Atemnotsyndrom und folgendem Nierenversagen vorstellte und im Verlauf der Erkrankung bereits im Juni 2012 verstarb. Die präsentierte Klinik zeigte sich hierbei vergleichbar dem Verlauf der schweren SARS-Erkrankungen aus dem Pandemiegeschehen des Jahres 2003. Auch bei hCoVEMC handelt es sich um ein Coronavirus mit genetischer Ähnlichkeit zu bekannten tierischen Fledermaus-Viren, welches bis zum heutigen Tag in 9 Fällen ernster Erkrankungsverläufe (5 Tote bislang) beim Menschen nachgewiesen werden konnte.

Der folgende Artikel soll einen kurzen Überblick zum aktuellen Stand des Wissens zu diesem neuen Coronavirus sowie Informationen zur Falldefinition und Meldung von Verdachtsfällen bzw. Erkrankungen in Deutschland geben (laut RKI).

\section{Infections with new human Betacoronavirus 2c EMC/2012 (hCoV-EMC)}

S. Uibel, D. A. Groneberg: Infections with new human Betacoronavirus 2c EMC/2012 (hCoV-EMC). Zbl Arbeitsmed 63 (2013) 54-56

Key words: $h$ CoV-EMC, Betacoronavirus 2c EMC/2012, human Coronavirus

\section{Summary:}

An unknown Coronavirus (hCoV-EMC) was first isolated retrospectively in September 2012 from sputum of a 60 year old Saudi Arabian patient, who suffered from acute respiratory failure followed by renal failure and died already in June 2012. The clinical findings were highly similar to those presented in the severe diseases seen in the SARS pandemic in 2003. Also the hCoV-EMC is genetically related to animal Coronaviruses (in bats) and caused 9 affirmed severe human infections leading to 5 deaths by now.

The following article should give a short overview concerning the current knowledge about the new human Coronavirus as well as information about the official disease definition and registration procedures in Germany (Robert Koch-Institut).

\section{Stand des Wissens zu hCoV-EMC}

Coronaviren kommen als Verursacher sehr unterschiedlich verlaufender Erkrankungen bei Vögeln, Säugetieren und dem Menschen vor. Die Bandbreite der menschlichen Erkrankungen reicht dabei von gastrointestinalen Beschwerden, Lebererkrankungen und neurologischen Symptomen über leichte bis zu foudroyant verlaufenden respiratorischen Infektionen (SARS) (Anderson and Baric 2012; WHO 2012). Coronaviren besitzen eine einzelsträngige RNA, welche eine hohe Rekombinationsfrequenz auf- weist und daher zu einer enormen Diversität der unterschiedlichen Viren beiträgt, was wiederum deren Fähigkeit begünstigt, sich schnell an neue Wirte, Umgebungen und ökologische Nischen anzupassen und damit auch Zoonosen auslösen zu können (Zaki, van Boheemen et al. 2012). Das nachgewiesene neue humane Betacoronavirus hCoVEMC hat genetisch seine nächste bekannte Verwandtschaft zu den Fledermaus-Coronaviren HKU4 und HKU5 (Chan, Li et al. 2012; Zaki, van Bohee- men et al. 2012). Aktuelle Zellkulturversuche des mittlerweile genetisch erfassten Virus zeigen eine hohe Anpassungsfähigkeit, da es zwischen menschlichen und tierischen Zellen problemlos zu wechseln vermag (Blawat 2012; van Boheemen, de Graaf et al. 2012). Weiterhin konnte bewiesen werden, dass hCoVEMC nicht die gleichen zellulären Zielstrukturen wie das SARS-Coronavirus verwendet - über welchen exakten Pathomechanismus es die Wirtszellen befällt, ist jedoch zum jetzigen Zeitpunkt

Die Autoren:

Stefanie Uibel $\mathbf{\square}$ David A. Groneberg

Institut für Arbeitsmedizin, Sozialmedizin und Umweltmedizin der Goethe-Universität Frankfurt am Main

Kontakt:vibel@med.uni-frankfurt.de 
noch nicht abschließend geklärt (Jiang, Lu et al. 2012; Muller, Raj et al. 2012).

\section{Erkrankungen und Epidemiologie}

Bislang sind unter akuten respiratorischen Infektionen 9 Fälle in Zusammenhang mit HCoV-EMC bestätigt. Die WHO vermutet, dass Infektionen $a b$ April 2012 aufgetreten sind, denn $\mathrm{HCoV}$-EMC wurde retrospektiv in zwei Fällen nachgewiesen in einer Gruppe von 11 epidemiologisch verbundenen Fällen akuter respiratorischer Infektionen in Jordanien, wovon 8 Personen in medizinischen Berufen arbeiteten (Corman, Muller et al. 2012).

Alle bestätigten Erkrankungsfälle wiesen einen schweren Krankheitsverlauf auf, fünf Patienten starben bisher an den Infektionen (RKI 2012a).

Eine detaillierte Fall- und Klinikbeschreibung des 60jährigen Saudi-Arabischen Patienten aus dem Juni 2012, bei dem erstmals das neue Coronavirus nachgewiesen werden konnte, stellten Zaki et al. im Oktober 2012 im New England Journal of Medicine vor (Zaki, van Boheemen et al. 2012).

In Jordanien traten zwei Todesfälle auf, in Saudi-Arabien erkrankten fünf Menschen, wovon drei starben. Drei dieser fünf Fälle, darunter zwei Todesfälle, gehörten hierbei zu einer familiären Häufung (RKI 2012a). Von den zwei in Katar erkrankten Patienten wurde einer in einer Essener Lungenklinik behandelt und Mitte November 2012 entlassen, der andere Patient ist noch aktuell in England in Behandlung und auf dem Weg der Besserung (Bermingham, Chand et al. 2012; RKI 2012a).

Es existieren noch keine epidemiologisch belastbaren Angaben zu den tatsächlichen Erkrankungshäufigkeiten sowie deren Letalität. Unter den momentan erfassten Erkrankungen zeigt sich allein eine Häufung auf der Arabischen Halbinsel. Ebenso gehen die Forschungen noch weiter, um die tatsächlichen Infektionsquellen und Übertragungswege $\mathrm{zu}$ identifizieren, wozu vorerst das Umfeld der bestätigten Krankheitsfälle untersucht wird (WHO 2012). Durch die clusterartige Verteilung der Erkrankungen kann eine Mensch-zu-Mensch-Übertragung nicht ausgeschlossen werden, allerdings könnte auch eine gemeinsame externe Quelle ursächlich sein (epidemiologische Verbindung zwischen den Fällen in SaudiArabien und Jordanien wurden bestätigt) (RKI 2012a).

\section{Nachweisverfahren}

Aktuell liegen zur schnellen Detektion von hCoV-EMC ein RT-PCR-Verfahren vor, sowie das sogenannte upE Gene Assay, wofür mehr als 100 Labore weltweit mit positivem Kontrollmaterial ausgestattet wurden, um den upE Assay durchführen zu können (Corman, Muller et al. 2012; Corman, Eckerle et al. 2012).

\section{Was ist in Deutschland derzeit gültig bzw. empfohlen?}

Da mögliche Infektionsquellen und Übertragungswege des neuen humanen Betacoronavirus 2c EMC/2012 noch nicht bekannt sind, rät das Robert Koch Institut die Versorgung von Patienten wahrscheinlicher bzw. bestätigter Fälle unter Einhaltung strenger Hygienemaßnahmen entsprechend den Empfehlungen für Erkrankungen durch das SARS-Virus zu realisieren (RKI 2012a).

Zur Fallfindung hat das Robert KochInstitut eine Falldefinition online gestellt (RKI 2012b), die hier jeweils aktualisiert abgerufen werden kann (momentan Stand 12.12.2012):

\section{http://www.rki.de/DE/Content/}

InfAZ/C/Corona/Corona_Falldefinition. pdf? _ blob=publicationFile

In dieser wird neben Krankheitssymptomen des Vorliegens akuter Pneumonien bzw. akuter Atemnotsyndrome auch explizit die epidemiologische Exposition innerhalb der letzten 10 Tage vor Krankheitsbeginn abgefragt: Neben einerseits engem Kontakt bei der Versorgung eines Erkrankten, sollen auch Aufenthalte auf der Arabischen Halbinsel sowie angrenzenden Ländern erfragt werden. Hierzu gehören (Stand 07.12.2012):

Jemen, Katar, Kuwait, Oman, SaudiArabien, Vereinigte Arabische Emirate sowie Irak, Jordanien, Bahrain, Syrien, Libanon, Iran, Palästinensische Gebiete, Israel.

Reisewarnungen aus infektiologischen Gründen bzgl. HCoV-EMC in die oben genannten Länder bestehen momentan nicht.

Ebenso wurde eine Meldepflicht eingeführt, nach der Verdachtsfälle (Patienten unter weiterer Abklärung), wahrscheinliche Fälle und bestätigte Fälle entsprechend der RKI-Falldefinition wegen der Schwere der Erkrankungen auf Grundlage von $\S 6$ Abs. 1 Nr. 5a IfSG (Auftreten einer bedrohlichen Krankheit, wenn dies auf eine schwerwiegende Gefahr für die Allgemeinheit hinweist und Krankheitserreger als Ursache in Betracht kommen, die nicht in $\S 7$ genannt sind) namentlich an das zuständige Gesundheitsamt zu melden sind. Darüber hinaus stellt das Gesundheitsamt gemäß $\S 25$ Abs. 1 IfSG ggf. eigene Ermittlungen an (RKI 2012a; RKI 2012b).

\section{Literatur}

Anderson, L. J. and R. S. Baric (2012). „Emerging human coronaviruses--disease potential and preparedness.“ N Engl J Med 367(19): 1850-1852.

Bermingham, A., M. A. Chand, et al. (2012). „Severe respiratory illness caused by a novel coronavirus, in a patient transferred to the United Kingdom from the Middle East, September 2012." Euro Surveill 17(40): 20290.

Blawat, K. (2012). „Neues Coronavirus - Forscher rätseln über seine Gefährlichkeit (17.12.2012).“ Retrieved 03.01.2013, from http://www.sueddeutsche.de/gesundheit/ neues-coronavirus-forscher-raetseln-ueberseine-gefaehrlichkeit-1.1552492.

Chan, J. F., K. S. Li, et al. (2012). „Is the discovery of the novel human betacoronavirus $2 \mathrm{c}$ EMC/2012 (HCoV-EMC) the beginning of another SARS-like pandemic?"“ J Infect 65(6): 477-489.

Corman, V., M. Muller, et al. (2012). „Assays for laboratory confirmation of novel human coronavirus (hCoV-EMC) infections." Euro Surveill 17(49).

Corman, V. M., I. Eckerle, et al. (2012). „Detection of a novel human coronavirus by real-time reverse-transcription polymerase chain reaction." Euro Surveill 17(39).

Jiang, S., L. Lu, et al. (2012). „A predicted receptor-binding and critical neutralizing domain in $\mathrm{S}$ protein of the novel human coronavirus HCoV-EMC." J Infect.

Muller, M. A., V. S. Raj, et al. (2012). „Human Coronavirus EMC Does Not Require the SARS-Coronavirus Receptor and Maintains Broad Replicative Capability in Mammalian Cell Lines.“" MBio 3(6).

RKI (2012a). „Epidemiologisches Bulletin Nr. 49 vom 10. Dezember 2012."S. 494.

RKI (2012b). ,Schwere respiratorische Erkrankungen in Verbindung mit einem neuartigen Coronavirus. Falldefinition zur Fallfindung, Meldung und Übermittlung (12.12.2012).“ 
van Boheemen, S., M. de Graaf, et al. (2012). „Genomic characterization of a newly discovered coronavirus associated with acute respiratory distress syndrome in humans." MBio 3(6). WHO. (2012). „Background and summary of novel coronavirus infection - as of 30 November 2012." Retrieved 03.01.2013, from http://www.who.int/csr/disease/coronavi rus_infections/update_20121130/en/.

Zaki, A. M., S. van Boheemen, et al. (2012) „Isolation of a novel coronavirus from a man with pneumonia in Saudi Arabia." N Engl J Med 367(19): 1814-1820.

\author{
Abkürzungen: \\ RKI Robert Koch-Institut \\ RT-PCR Real-Time Reverse Transkriptase \\ Polymerase-Ketten-Reaktion \\ SARS Schweres Akutes Respiratorisches \\ Syndrom \\ WHO World Health Organization
}

\section{Aus den Akademien}

\section{Sozial- und Arbeitsmedizinische Akademie Baden-} Württemberg e.V. in Verbindung mit der Universität Ulm

Weiterbildungskurse Arbeitsmedizin/Betriebsmedizin Kurs B $\square$ Teil 1 in Ulm $\square 21.02 .2013-01.03 .2013$ Kurs B $\square$ Teil 2 in Ulm $\square$ 05.03.2013-12.03.2013

\section{Anmeldungen für alle Kurse bitte an die}

Sozial- und Arbeitsmedizinische Akademie Baden-Württemberg e.V. - Geschäftsstelle Ulm a Oberer Eselsberg 45 - 89081 Ulm

- Tel. 0731/54044 a Fax: 0731/552642 — E-Mail: ulm@samanet.de - www.sama.de

\section{Bayerische Akademie für Arbeits-, Sozial- und Umweltmedizin}

Kursinhalte Arbeitsmedizin/Betriebsmedizin B-Kurs: 10.06.-28.06.2013 — C-Kurs: 07.10.-25.10.2013

\section{Weitere Informationen:}

www.lgl.bayern.de/aus_fort_weiterbildung/weiterbildung/ arbeitsmedizin/index.htm

\section{Ärztekammer Berlin}

Abteilung Fortbildung / Qualitätssicherung Akademie für Arbeitsmedizin und Gesundheitsschutz

Weiterbildungskurs „Arbeitsmedizin / Betriebsmedizin“ Kursteil A 1: 02.09. - 11.09.2013 — Kursteil A 2: 11.09. - 20.09.2013 Kursteil B 1: 04.11 - 13.11.2013 — Kursteil B 2: 13.11 - 22.11.2013 Kursteil C 1: 06.01 - 15.01.2014 — Kursteil C 2: 15.01 - 24.01.2014

\section{Weitere Informationen:}

Anmeldungen bitte an die Ärztekammer Berlin a Abteilung Fortbildung / Qualitätssicherung - Servicebereich AAG — Friedrichstraße 16 - 10969 Berlin — Tel.: (030) 40806 - 1215 - Fax: (030) 40806 - 55 1399 - E-Mail: fb-aag@aekb.de - www.aerztekammer-berlin.de

\section{Akademie für Ärztliche Fortbildung und Weiterbildung der Landesärztekammer Hessen}

Arbeits- und Betriebsmedizin

Theoretische Weiterbildung zum Erwerb der Gebietsbezeichnung Arbeitsmedizin bzw. Zusatzbezeichnung Betriebsmedizin Leitung: Prof. Dr. med. Dr. h. c. mult. D. Groneberg Prof. Dr. med. H.-J. Woitowitz

Termine: B1 16.03.-23.03.2013 — C1 13.04.-20.04 2013

- A2 14.09.-21.09.2013 — B2 26.10.-02.11.2013

- C2 30.11. - 07.12.2013 Nach den geltenden Bestimmungen der Ärztekammern müssen die jeweils zusammengehörenden Kursabschnitte (z.B. A1 u. A2) an der gleichen Akademie absolviert werden. Auskunft und Anmeldung: Akademie für Ärztliche Fortbildung und Weiterbildung der Landesärztekammer Hessen $\square$ Frau Luise Stieler - Carl-Oelemann-Weg 7 a 61231 Bad Nauheim — Fon: 06032 782-283 - Fax: 069 97672-67283 E- E-Mail: Luise.Stieler@laekh.de - www.fbz-hessen.de

\section{Academic Education and training}

\section{Nordrheinische Akademie für ärztliche Fort- und} Weiterbildung

Ärztekammer Nordrhein/Kassenärztliche Vereinigung Nordrhein

\section{Kurse Arbeitsmedizin}

Teil A2 25.02.2013-06.03.2013

Teil B1 08.04.2013-17.04.2013 — Teil B2 08.07.2013-17.07.2013

Teil C1 02.09.2013-11.09.2013 — Teil C2 11.11.2013-20.11.2013

\section{Weitere Informationen:}

Referenten: Dipl.-Min. Silvester Siegmann $~$ Prof. Dr. med. Elisabeth Borsch-Galetke Dr. med. Klaus Siegmund

Ort: Institut für Arbeitsmedizin und Sozialmedizin

- Heinrich-Heine-Universität Düsseldorf — Gebäude 23.11

- Universitätsstr. 1 - D-40225 Düsseldorf Anmeldung: Nordrheinische Akademie für ärztliche Fort- und Weiterbildung Herr Norbert

Dohm — Tel.: 0211 4302-2831 — Anmeldung: n.dohm@aekno.de

\section{Sächsische Landesärztekammer}

Weiterbildungskurse Arbeitsmedizin/Betriebsmedizin 2013 der Sächsischen Akademie für ärztliche Fort- und Weiterbildung

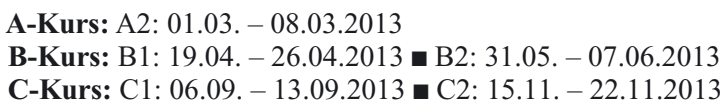

\section{Weitere Informationen:}

Wissenschaftliche Leitung: Prof. Dr. med. Andreas Seidler (wiss. Leiter) - Institut und Poliklinik für Arbeits- und Sozialmedizin der TU Dresden - Fetscherstr. 74 @ 01307 Dresden — Tel. (0351) 3177441

- E-Mail: eva.aris@mailbox.tu-dresden.de

Dr. med. Giso Schmeißer (stellv. wiss. Leiter) — Tel. (0351) 4571551 - E-Mail: Giso.Schmeisser@dguv.de — Veranstalter: Sächsische Landesärztekammer — Schützenhöhe 16 — 01099 Dresden - E-Mail: Kurse@SLAEK.de — Veranstaltungsort: Schützenhöhe 16 • 01099 Dresden — Tel. 03518267324 • Fax 03518267322

Akademie für ärztliche Fortbildung der Ärztekammer Westfalen-Lippe und der Kassenärztlichen Vereinigung Westfalen-Lippe

Weiterbildung zum Arbeitsmediziner/Betriebsmediziner in Bochum Kursteil B: B2: 25.02.2013-06.03.2013 Kursteil C: C1: 15.04.2013-24.04.2013 — C2: 03.06.-12.06.2013

Ort: Bochum - Seminarbereich des IPA Raum 10.03D.070 - Bürkle-de-la-Camp-Platz 1

Gesamtleitung: Prof. Dr. med. Thomas Brüning Gesamtorganisation: Dr. med. Horst Christoph Broding

Weitere Informationen:

Ansprechpartnerin: Frau Anja Huster $\square$ Tel.: 0251/929-2202 — Schriftliche Anmeldung erforderlich an: Akademie für ärztliche Fortbildung der ÄKWL und der KVWL — Postfach 4067 — 48022 Münster - Fax: 0251/929-2249 